\title{
Flexo-photovoltaic effect
}

\author{
Ming-Min Yang, ${ }^{*}$ Dong Jik Kim, ${ }^{*}$ Marin Alexe† \\ Department of Physics, University of Warwick, Coventry, CV4 7AL, UK. \\ *These authors contributed equally to this work. \\ †Corresponding author. Email: m.alexe@warwick.ac.uk
}

It is highly desirable to discover photovoltaic mechanisms that enable a higher efficiency of solar cells. Here, we report that the bulk photovoltaic effect, which is free from the thermodynamic ShockleyQueisser limit but usually manifested only in noncentrosymmetric (piezoelectric or ferroelectric) materials, can be realized in any semiconductor, including silicon, by mediation of flexoelectric effect. We introduce strain gradients using either an atomic force microscope or a micron-scale indentation system, creating giant photovoltaic currents from centrosymmetric single crystals of $\mathrm{SrTiO}_{3}$, $\mathrm{TiO}_{2}$, and $\mathrm{Si}$. This strain-gradient-induced bulk photovoltaic effect, which we call the flexo-photovoltaic effect, functions in the absence of a $p$ - $n$ junction. This finding may extend present solar cell technologies by boosting the solar energy conversion efficiency from a wide pool of established semiconductors.

Since its first observation in the nineteenth century, the photovoltaic (PV) effect has been studied intensively for scientific interest and as a sustainable energy source to replace fossil fuels and reduce carbon emissions (1-3). In 1954, the first high-power modern silicon solar cells were invented in which the photoexcited carriers were separated by a built-in electrical field developed at a $p$ - $n$ junction (4). By virtue of the appropriate bandgap energies of the semiconductors in a $p$ - $n$ junction solar cell, sunlight is efficiently absorbed, resulting in considerable power conversion efficiency. Nowadays, silicon PV cells are the mainstay of the modern solar industry, contributing more than $1 \%$ of global electricity supply. Nevertheless, PV cells based on $p$ - $n$ junctions have a photovoltage limited by the bandgap energy of the constituent semiconductors and an ultimate efficiency constrained by the Shockley-Queisser (SQ) limit (5).

The ferroelectric materials exhibit a photovoltaic effect distinct from that of $p$ - $n$ junctions, called the bulk photovoltaic (BPV) effect $(6,7)$. Under uniform illumination, a homogeneous ferroelectric material gives rise to a current under zero bias (short-circuit current; $I_{S C}$ ) that depends on the polarization state of the incident light and produces an anomalously large photovoltage well exceeding its bandgap energy. This peculiar PV effect originates from the asymmetric distribution of photoexcited nonequilibrium carriers in $k$-space caused by the absence of centrosymmetry in the material (7). Owing to its distinctive charge separation mechanism, solar cells based on the BPV effect can in principle exceed the S-Q limit (8). However, a substantial BPV effect is generally found in wide-bandgap noncentrosymmetric materials, such as ferroelectric $\mathrm{BaTiO}_{3}(8)$ and $\mathrm{BiFeO}_{3}(9)$, leading to an overall extremely low device efficiency under solar illumination.
Hence, one way to enhance the efficiency may be to realize the BPV effect in semiconductors with more favorable bandgaps, regardless of their crystalline symmetry.

Flexoelectricity is an electromechanical property that reflects a coupling between an electric polarization and a strain gradient $(10,11)$. In centrosymmetric materials, a strain gradient breaks its inversion symmetry, resulting in a polarization with a preferred direction and enabling a piezoelectric composite containing no piezoelectric elements $(11,12)$. Likewise, one can hypothesize that a strain gradient allows the manifestation of the BPV effect in materials that are originally centrosymmetric. Here, we propose and demonstrate that the BPV effect can be induced in any semiconductor by mediation of the flexoelectric effect. Given that flexoelectricity is a universal property of all materials, ranging from biomaterials (13) to semiconductors and dielectrics (14) to 2D materials such as graphene (15), this strain-gradient-induced BPV effect, termed here the flexo-photovoltaic (FPV) effect, is allowed for all symmetry classes. Thus, the devices based on the FPV effect can be fabricated with silicon or any other semiconductors.

To demonstrate our idea, we explored the PV effect induced by a point force exerted onto the surface of centrosymmetric materials including a $\mathrm{SrTiO}_{3}$ single crystal and a rutile $\mathrm{TiO}_{2}$ single crystal (see section $\mathrm{S} 1$ of (16)). The point force was exerted by the tip of an atomic force microscope (AFM), inducing local inhomogeneous strain at the tip/surface contact area and, therefore, a local breaking of centrosymmetry (17, 18). In our experiments, we used a custom-made photoelectric atomic force microscope (Ph-AFM) consisting of an AFMbased system equipped with a customized current amplifier/filter system and an optical system (19). The optical system allows us to illuminate a sample surface with a 405-nm 
laser properly polarized by a half-wavelength plate. A conductive AFM tip applies a local force on a sample surface and simultaneously collects the resultant PV current. A brief schematic of the Ph-AFM setup is shown in Fig. 1, A and B.

$\mathrm{SrTiO}_{3}$ single crystal is one of the ideal systems to study the flexoelectric effect thanks to its simple cubic centrosymmetric lattice and large dielectric permittivity $(14,20)$. Unlike its sister material $\mathrm{BaTiO}_{3}$, ordinary $\mathrm{SrTiO}_{3}$ crystals do not exhibit the BPV effect because they have a center of inversion symmetry. But, with illumination around the contact area on a (001)-face of $\mathrm{SrTiO}_{3}$ crystal, we observed that $I_{S C}$ exhibits a large transient peak as the loading force is increased from 1 $\mu \mathrm{N}$ to $18 \mu \mathrm{N}$ (Fig. 1C), which is reproducible, proven by repeated exertion/withdrawal of the loading force.

To show that the giant enhancement of $I_{S C}$ by the point force is not confined to $\mathrm{SrTiO}_{3}$ crystals or a cubic structure, we investigated the force-induced PV current in a single crystal of rutile $\mathrm{TiO}_{2}$, which is well-known for its photoelectric applications such as dye-sensitized photovoltaic cells and photocatalysis. As in the case of $\mathrm{SrTiO}_{3}$, large negative $I_{S C}$ appears once a large force is exerted on $\mathrm{TiO}_{2}$ (100) face. Figure 1D shows a stable current under the $15 \mu \mathrm{N}$ force, confirming that the force-induced PV effect is not a transient effect in this material. Although it depends on contact conditions and locations, the point force exerted by the AFM tip gives rise to a substantial current density up to about $1 \mathrm{~A} / \mathrm{cm}^{2}$ at the nanoscale contact area. This is over three orders of magnitude higher than $I_{S C}\left(0.3 \mathrm{~mA} / \mathrm{cm}^{2}\right)$ obtained from a Schottky junction between $\mathrm{TiO}_{2}$ and $\mathrm{Pt}$ under the same illumination condition (see section S6 of (16)).

The current density increases more than 100 times by simply increasing the loading force from 1 to $15 \mu \mathrm{N}$ (Fig. 1E). This cannot be explained with an increase of the contact area with the loading force because a contact area increases at most $\sim 6$ times with a 15 times larger contact force in a simple elastic sphere contact model (see section S2 of (16)). Moreover, a 100 times increase of the contact area with an AFM tip is unphysical. It should be note that $I_{S C}$ turns to a positive value when loading the conductive AFM tip on a (001)-face of the $\mathrm{TiO}_{2}$ crystal in contrast to the negative value of $I_{S C}$ on its (100)-face (Fig. 1F). The fact that the direction of the PV current depends on the crystallographic orientation of the $\mathrm{TiO}_{2}$ crystal indicates that the observed PV effect cannot be merely attributed to a probable Schottky contact formed by the $\mathrm{TiO}_{2}$ surface and the Pt-coating of the AFM tip.

A potential origin of this $\mathrm{PV}$ current enhancement is the BPV effect. As hypothesized, it is likely that a point force exerted on a crystal surface generates a local strain gradient, resulting in local centrosymmetry breaking and, thus, a local BPV current under illumination, i.e., the FPV effect, in the absence of a $p$ - $n$ junction and an appropriate band alignment. Given that both the flexoelectric response and the BPV effect depend on the crystallographic orientation (19-21), our results suggest a predominant role of the strain gradient and the resultant FPV effect in the enhanced local PV current.

The strain gradient induced by a sphere contact has a complex spatial distribution with giant strain gradient values in an elastic material, as described in section S2 of (16). An AFM tip apex can be approximated as a hemisphere and the distribution of strain gradient induced by the AFM tip can be calculated analytically with the Hertzian model and the Boussinesq's calculation (22). Figure 2 shows the spatial distributions of the z-component of the calculated strain and its derivative with respect to $z$ under $\sim 15.7 \mu \mathrm{N}$ force with a 10nm-radius contact area at the origin. The strain gradient is as large as $10^{7} \mathrm{~m}^{-1}$. Interestingly, we found that the $I_{S C}$ density and the volume beneath the AFM tip being subject to a strain gradient larger than $1 \times 10^{6} \mathrm{~m}^{-1}$ show similar dependence on the exerted force (Fig. 1E); the detailed process to obtain the relative volume can be found in fig. S9 of (16)). It is expected that the giant strain gradients induced by the AFM tip break local symmetry, leading to the manifestation of the BPV effect locally under illumination. However, a relationship between the complex distribution of the strain gradient and the BPV properties should be quite intricate and deep theoretical considerations are required.

The main characteristic of the BPV effect is a periodic dependence on the angle between the PV current and the light polarization, stemming from its tensorial nature (21). For the present case, this dependence is predicted to be

$$
I_{F P V}=\frac{\pi}{2} I_{0}\left(A_{z}+B_{z} \cos 2 \alpha\right)
$$

Where $I_{\mathrm{FPV}}$ is the PV current, $I_{0}$ is a light intensity, $A_{Z}$ and $B_{Z}$ are effective BPV coefficients of the locally deformed crystal, and $\alpha$ is the polarization angle of the incident light with respect to the top surface edge as described in section S3 of (16). The FPV effect should inherit the distinctive feature of the BPV effect and exhibit a sinusoidal dependence on the incident light polarization angle with a period of $180^{\circ}$. Indeed, $I_{S C}$ measured by a conductive AFM tip in the configuration illustrated in Fig. 1B on the $\mathrm{SrTiO}_{3}(010)$ and $\mathrm{TiO}_{2}(001)$ surfaces exhibits a light polarization dependence in accord with Eq. 1, as seen in Fig. 3. The sinusoidal behavior upon rotating the light polarization provides strong evidence that the underlying mechanism of the force-induced PV effect is the BPV effect generated by local symmetry breaking due to inhomogeneous strain, namely the FPV effect.

The FPV effect should be neither confined to ionic crystals nor restricted to nanoscale geometries. First, we performed the same experiment (as in Fig. 1) on an HF-passivated surface of a commercial $p$-type Si (001) crystal. Similarly, $I_{S C}$ increased two orders of magnitude from $\sim 5 \mathrm{pA}$ with the $1 \mu \mathrm{N}$ loading force to $\sim 0.5 \mathrm{nA}$ with the $15 \mu \mathrm{N}$ loading force (Fig. 4A and section S7 of (16)). Secondly, the FPV effect could also be 
demonstrated using a home-built indentation system that deforms a semiconductor using a conductive tungsten probe needle with a radius of about $10 \mu \mathrm{m}$ (fig. S1 of (16)). Figure $4 \mathrm{~B}$ shows crystallographic orientation dependent photocurrent-voltage $(I-V)$ characteristics of the $\mathrm{SrTiO}_{3}$ crystal acquired under $4 \mathrm{~N}$ mechanical force exerted by the probe needle and $405 \mathrm{~nm}$ laser illumination directly to the probe contact area as in Fig. 1A. These linear $I-V$ characteristics are similar to those of ferroelectrics under illumination (23). The oscillating PV current with the rotating light polarization, which is well fitted with Eq. 1 (Fig. 4C), demonstrates the FPV effect under the indentation force in the micrometer scale. The crystallographic orientation dependent PV current is also observed on the $\mathrm{TiO}_{2}$ crystal by the indentation system (fig. S2 of (16)). The persistence of the FPV effect from the nanoscale (AFM) to the micrometer level is promising for future device design and potential applications. The FPV effect is not related to a plasmonic effect found in the tip-enhanced Raman scattering because only an atomically sharp tip coated with $\mathrm{Ag}$ or $\mathrm{Au}$ shows the plasmonic enhancement in the visible range (24), whereas we used platinum or tungsten probes ranging from the nanoscale to the micrometer scale. Likewise, a potential cubic-to-tetragonal phase transition induced in a $\mathrm{SrTiO}_{3}$ single crystal under a large hydrostatic pressure ( $>6 \mathrm{GPa}$ ) should not play a large role here because of the centrosymmetric nature of the induced tetragonal phase (25).

We emphasize here four main features of the FPV effect. First, the separation of the photoexcited carriers in the FPV effect is controlled by the local symmetry and the resultant local BPV effect, in which the power conversion efficiency can in principle exceed the S-Q limit (8). Second, to obtain a high photocurrent from any semiconductor, only a strain gradient generator, such as a sharp probe with a sufficient loading force is necessary. This should be distinguished from the previous reports that a strain gradient modifies a bandgap, but the charge separation in the photoelectric process still requires a proper band alignment (26) or a nanostructure (27, 28 ). Third, whereas the BPV effect is allowed only in non-centrosymmetric materials, the FPV effect is universal. It is allowed by symmetry in all materials thanks to the universal nature of the strain-gradient-induced centrosymmetry breaking. The FPV effect can be realized not only in ionic crystals $\left(\mathrm{SrTiO}_{3}\right.$ and $\left.\mathrm{TiO}_{2}\right)$ and covalent crystals ( $\left.\mathrm{Si}\right)$, but is also relevant to any semiconductors ranging from organic-inorganic hybrid perovskites to semiconducting polymers and even topological insulators. For instance, the topological insulator $\mathrm{Bi}_{2} \mathrm{Te}_{3}$ with a centrosymmetric structure exhibits the BPV effect by means of the flexoelectric effect (29). Finally, given that the BPV effect consists of asymmetric quantum mechanical processes such as photoexcitation, relaxation, recombination, and scattering, we demonstrate that one can readily control the quantum mechanical processes by macroscopic tool such as an AFM tip and a probe needle.

The configuration of our photovoltaic indentation system is very simple and the FPV effect can be increasingly significant with material dimensions decreasing into the nanoscale where flexoelectricity is more important (11). Thus, an important strain engineering route for improving the final performance of solar cells and optoelectronic devices is now open. For example, a tandem solar cell can be easily fabricated by combining an array of indenters and a conventional $p$ - $n$ junction, enabling a higher efficiency because the FPV effect can be designed to add to the existing PV current generated by the buried $p$ - $n$ junction. Given that the lattice mismatch at the interfaces and crystallographic disorders in epitaxial and polycrystalline thin film solar cells give rise to substantial strain gradients (30-32), the associated FPV effect would dramatically impact the performance of these solar cells, which however remains largely unexplored. In addition to inorganic solar cells, the FPV effect is also likely to play an important role in flexible and stretchable electronics based on organic and polymeric semiconductors. Not only the bending of flexible organic devices at a macroscopic level (33) but also the folding and entanglement of the polymeric chains in a nanoscale level would generate sizable strain gradients (34), which redistribute the electron cloud of $\pi$-molecular orbits, modifying the electronic transport and inducing the FPV effect under illumination $(15,35)$. The detailed effects of strain gradient and FPV effect on the nanoscale electronic properties remain an open question.

\section{REFERENCES AND NOTES}

1. W. Siemens, On the Electromotive Action of Illuminated Selenium, Discovered by Mr. Fritts of New York. Lond. Edinb. Dublin Philos. Mag. J. Sci. 19, 315-316 (1885). doi:10.1080/14786448508627681

2. M. A. Green, S. P. Bremner, Energy conversion approaches and materials for highefficiency photovoltaics. Nat. Mater. 16, 23-34 (2017). doi:10.1038/nmat4676 Medline

3. A. Polman, M. Knight, E. C. Garnett, B. Ehrler, W. C. Sinke, Photovoltaic materials: Present efficiencies and future challenges. Science 352, aad4424 (2016). doi:10.1126/science.aad4424 Medline

4. A. Fahrenbruch, R. Bube, Fundamentals of Solar Cells: Photovoltaic Solar Energy Conversion (Elsevier, 2012).

5. W. Shockley, H. J. Queisser, Detailed balance limit of efficiency of $p-n$ junction solar cells. J. Appl. Phys. 32, 510-519 (1961). doi:10.1063/1.1736034

6. A. Chynoweth, Surface space-charge layers in barium titanate. Phys. Rev. 102 , 705-714 (1956). doi:10.1103/PhysRev.102.705

7. B. I. Sturman, V. M. Fridkin, The Photovoltaic and Photorefractive Effects in Noncentrosymmetric Materials (Gordon and Breach Science Publishers, 1992).

8. J. E. Spanier, V. M. Fridkin, A. M. Rappe, A. R. Akbashev, A. Polemi, Y. Qi, Z. Gu, S. M. Young, C. H. Hawley, D. Imbrenda, G. Xiao, A. L. Bennett-Jackson, C. L. Johnson, Power conversion efficiency exceeding the Shockley-Queisser limit in a ferroelectric insulator. Nat. Photonics 10, 611-616 (2016). doi:10.1038/nphoton.2016.143

9. A. Bhatnagar, A. R. Chaudhuri, Y. H. Kim, D. Hesse, M. Alexe, Role of domain walls in the abnormal photovoltaic effect in $\mathrm{BiFeO}_{3}$. Nat. Commun. 4, 2835 (2013). doi:10.1038/ncomms3835

10. A. K. Tagantsev, Piezoelectricity and flexoelectricity in crystalline dielectrics. Phys. Rev. B 34, 5883-5889 (1986). doi:10.1103/PhysRevB.34.5883 Medline 
11. P. Zubko, G. Catalan, A. K. Tagantsev, Flexoelectric effect in solids. Annu. Rev. Mater. Res. 43, 387-421 (2013). doi:10.1146/annurev-matsci-071312-121634

12. J. Fousek, L. Cross, D. Litvin, Possible piezoelectric composites based on the flexoelectric effect. Mater. Lett. 39, 287-291 (1999). doi:10.1016/S0167577X(99)00020-8

13. K. D. Breneman, W. E. Brownell, R. D. Rabbitt, Hair cell bundles: Flexoelectric motors of the inner ear. PLOS ONE 4, e5201 (2009). doi:10.1371/journal.pone.0005201 Medline

14. U. K. Bhaskar, N. Banerjee, A. Abdollahi, Z. Wang, D. G. Schlom, G. Rijnders, G. Catalan, A flexoelectric microelectromechanical system on silicon. Nat. Nanotechnol. 11, 263-266 (2016). doi:10.1038/nnano.2015.260 Medline

15. S. V. Kalinin, V. Meunier, Electronic flexoelectricity in low-dimensional systems. Phys. Rev. B 77, 033403 (2008). doi:10.1103/PhysRevB.77.033403

16. Supplementary Materials.

17. J. Očenášek, H. Lu, C. W. Bark, C. B. Eom, J. Alcalá, G. Catalan, A. Gruverman, Nanomechanics of flexoelectric switching. Phys. Rev. B 92, 035417 (2015). doi:10.1103/PhysRevB.92.035417

18. H. Lu, C.-W. Bark, D. Esque de los Ojos, J. Alcala, C. B. Eom, G. Catalan, A. Gruverman, Mechanical writing of ferroelectric polarization. Science 336, 59-61 (2012). doi:10.1126/science.1218693 Medline

19. M. M. Yang, A. Bhatnagar, Z. D. Luo, M. Alexe, Enhancement of local photovoltaic current at ferroelectric domain walls in $\mathrm{BiFeO}_{3}$. Sci. Rep. 7, 43070 (2017). doi:10.1038/srep43070 Medline

20. P. Zubko, G. Catalan, A. Buckley, P. R. Welche, J. F. Scott, Strain-gradient-induced polarization in $\mathrm{SrTiO}_{3}$ single crystals. Phys. Rev. Lett. 99, 167601 (2007). doi:10.1103/PhysRevLett.99.167601 Medline

21. V. Fridkin, Bulk photovoltaic effect in noncentrosymmetric crystals. Crystallogr. Rep. 46, 654-658 (2001). doi:10.1134/1.1387133

22. A. C. Fischer-Cripps, Introduction to Contact Mechanics (Springer, 2000).

23. V. M. Fridkin, Photoferroelectrics, vol. 9. (Springer Science \& Business Media, 2012).

24. M. D. Sonntag, E. A. Pozzi, N. Jiang, M. C. Hersam, R. P. Van Duyne, Recent advances in tip-enhanced Raman spectroscopy. J. Phys. Chem. Lett. 5, 31253130 (2014). doi:10.1021/jz5015746 Medline

25. M. Guennou, P. Bouvier, J. Kreisel, D. Machon, Pressure-temperature phase diagram of $\mathrm{SrTiO}_{3}$ up to $53 \mathrm{GPa}$. Phys. Rev. B 81, 054115 (2010). doi:10.1103/PhysRevB.81.054115

26. J. Feng, X. Qian, C.-W. Huang, J. Li, Strain-engineered artificial atom as a broadspectrum solar energy funnel. Nat. Photonics 6, 866-872 (2012). doi:10.1038/nphoton.2012.285

27. X. Fu, G. Jacopin, M. Shahmohammadi, R. Liu, M. Benameur, J.-D. Ganière, J. Feng, W. Guo, Z.-M. Liao, B. Deveaud, D. Yu, Exciton drift in semiconductors under uniform strain gradients: Application to bent ZnO microwires. ACS Nano 8, 34123420 (2014). doi:10.1021/nn4062353 Medline

28. J. Greil, S. Birner, E. Bertagnolli, A. Lugstein, Nanowire enabling strained photovoltaics. Appl. Phys. Lett. 104, 163901 (2014). doi:10.1063/1.4871458

29. Y. Liu, J. Chen, C. Wang, H. Deng, D.-M. Zhu, G. Hu, X. Chen, N. Dai, Bulk photovoltaic effect at infrared wavelength in strained $\mathrm{Bi}_{2} \mathrm{Te}_{3}$ films. APL Mater. 4 , 126104 (2016). doi:10.1063/1.4971798

30. M. S. Bennett, J. J. Kramer, The effect of piezoelectrically coupled stresses on the junction characteristics of CdS-Cu2S solar cells. J. Appl. Phys. 54, 7159-7165 (1983). doi:10.1063/1.331987

31. D. Lee, A. Yoon, S. Y. Jang, J.-G. Yoon, J.-S. Chung, M. Kim, J. F. Scott, T. W. Noh, Giant flexoelectric effect in ferroelectric epitaxial thin films. Phys. Rev. Lett. 107, 057602 (2011). doi:10.1103/PhysRevLett.107.057602 Medline

32. M.-W. Chu, I. Szafraniak, R. Scholz, C. Harnagea, D. Hesse, M. Alexe, U. Gösele, Impact of misfit dislocations on the polarization instability of epitaxial nanostructured ferroelectric perovskites. Nat. Mater. 3, 87-90 (2004). doi:10.1038/nmat1057 Medline

33. M. A. Reyes-Martinez, A. J. Crosby, A. L. Briseno, Rubrene crystal field-effect mobility modulation via conducting channel wrinkling. Nat. Commun. 6, 6948 (2015). doi:10.1038/ncomms7948 Medline
34. Q. Tang, Y. Tong, Y. Zheng, Y. He, Y. Zhang, H. Dong, W. Hu, T. Hassenkam, T. Bjørnholm, Organic nanowire crystals combine excellent device performance and mechanical flexibility. Small 7, 189-193 (2011). doi:10.1002/smll.201001217 Medline

35. T. Dumitrică, C. M. Landis, B. I. Yakobson, Curvature-induced polarization in carbon nanoshells. Chem. Phys. Lett. 360, 182-188 (2002). doi:10.1016/S00092614(02)00820-5

36. J. F. Nye, Physical Properties of Crystals: Their Representation by Tensors and Matrices (Oxford Univ. Press, 1985)

37. M.-M. Yang, Z.-D. Luo, D. J. Kim, M. Alexe, Bulk photovoltaic effect in monodomain $\mathrm{BiFeO}_{3}$ thin films. Appl. Phys. Lett. 110, 183902 (2017). doi:10.1063/1.4983032

38. R. H. Bube, Photoconductivity of Solids (Wiley, 1960).

39. A. M. Glass, D. von der Linde, T. J. Negran, High-voltage bulk photovoltaic effect and the photorefractive process in LiNb03. Appl. Phys. Lett. 25, 233-235 (1974). doi:10.1063/1.1655453

40. P. Gunter, Photovoltages, photocurrents and photorefractive effects in $\mathrm{KNbO}_{3}: \mathrm{Fe}$. Ferroelectrics 22, 671-674 (1978). doi:10.1080/00150197808237365

41. Z. Gu, D. Imbrenda, A. L. Bennett-Jackson, M. Falmbigl, A. Podpirka, T. C. Parker, D. Shreiber, M. P. Ivill, V. M. Fridkin, J. E. Spanier, Mesoscopic free path of nonthermalized photogenerated carriers in a ferroelectric insulator. Phys. Rev. Lett. 118, 096601 (2017). doi:10.1103/PhysRevLett.118.096601 Medline

42. M. Alexe, D. Hesse, Tip-enhanced photovoltaic effects in bismuth ferrite. Nat. Commun. 2, 256 (2011). doi:10.1038/ncomms1261

43. A. Zenkevich, Yu. Matveyev, K. Maksimova, R. Gaynutdinov, A. Tolstikhina, V. Fridkin, Giant bulk photovoltaic effect in thin ferroelectric $\mathrm{BaTiO}_{3}$ films. Phys. Rev. B 90, 161409 (2014). doi:10.1103/PhysRevB.90.161409

\section{ACKNOWLEDGMENTS}

Authors acknowledge Dr. James Lloyd-Hughes for the grammar revision of the manuscript and Mr. Binglin Tao for the support of schematic drawing. Funding: M.M.Y. acknowledges the University of Warwick for a Chancellor's International Scholarship. M.A. acknowledges the Wolfson Research Merit and Theo Murphy Blue-sky Awards of Royal Society. The work was partly supported by the EPSRC (UK) through grants no. EP/M022706/1, EP/P031544/1, and EP/P025803/1. Author contributions: D.J.K. initiated the project. M.-M.Y., D.J.K. and M.A. conceived and developed the ideas. M.-M.Y. and D.J.K. designed and conducted the experiments, analyzed the data, and wrote the manuscript. All authors contributed to the discussion of the results and the manuscript revision. M.A. supervised the project. Competing interests: M.-M.Y., D.J.K. and M.A. are authors of a British Patent Application No. GB1702466.2 filed in the UK by University of Warwick on 15/Feb/2017 that covers photovoltaic devices based on strain gradient inducer. Data and materials availability: All scientific data related to the present paper is available at University of Warwick open access research repository, http://wrap.warwick.ac.uk/100429.

\section{SUPPLEMENTARY MATERIALS}

www.sciencemag.org/cgi/content/full/science.aan3256/DC1

Supplementary Text

Figs. S1 to S15

References (36-43)

28 March 2017; accepted 6 April 2018

Published online 19 April 2018

10.1126/science.aan3256 
A
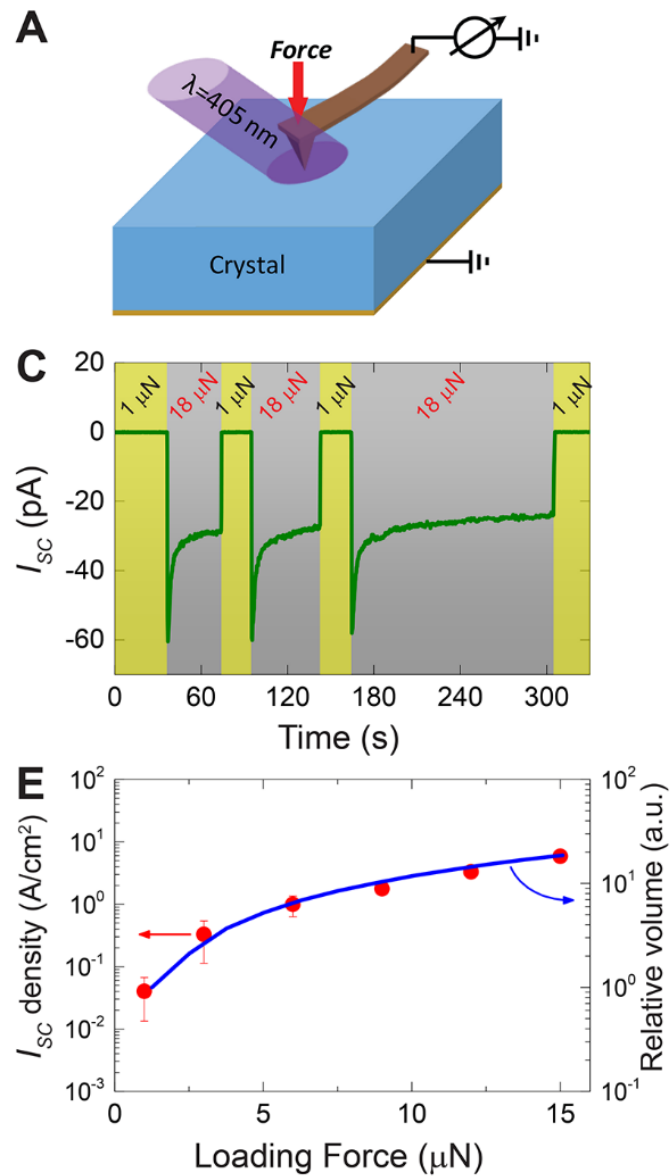
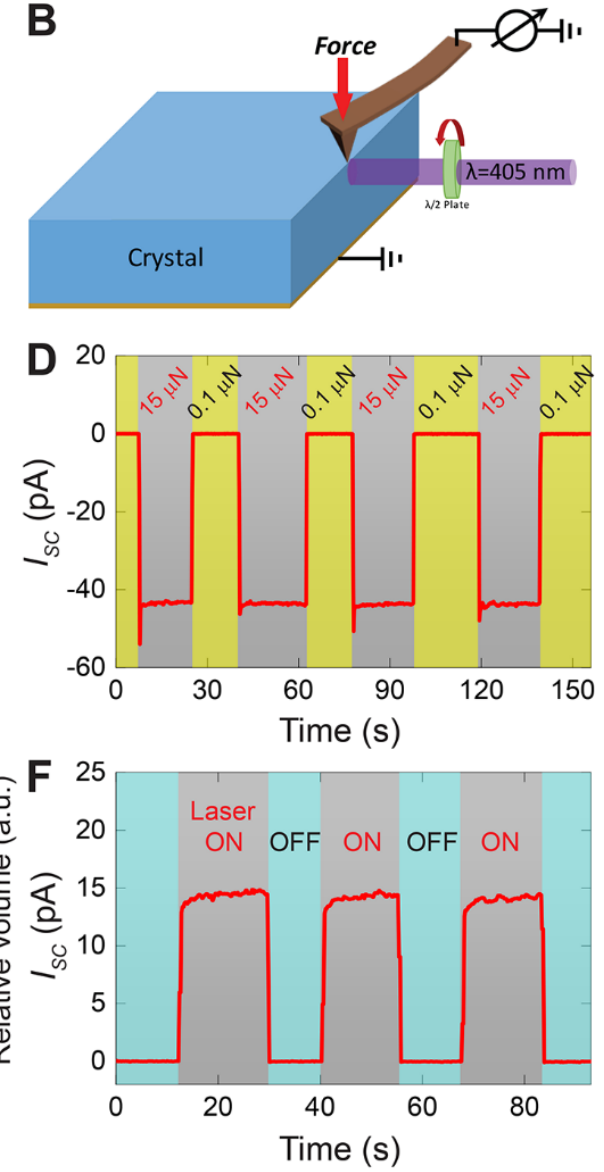

Fig. 1. Force-induced photovoltaic effect in centrosymmetric $\mathrm{SrTiO}_{3}$ and $\mathrm{TiO}_{2}$ single crystals. (A) Setup for illumination around the contact area. The tip loading force is controlled by the feedback loop of atomic force microscope. (B) Setup for illumination on the side surface. The sidewise illumination geometry has been chosen to avoid the effect of Fresnel reflection and have light absorption being independent of the light polarization. Evolution of the photocurrent induced and collected by a conductive AFM tip with a high loading force on (C) $\mathrm{SrTiO}_{3}(001)$ face and (D) $\mathrm{TiO}_{2}$ (100) face. (E) The loading force dependence of the induced photocurrent density and the relative volume subject to the strain gradient higher than $1 \times 10^{6} \mathrm{~m}^{-1}$ (see fig. S9 of (16)). (F) Positive photocurrent measured on $\mathrm{TiO}_{2}(001)$ face with a $15 \mu \mathrm{N}$ force applied by the AFM tip. 

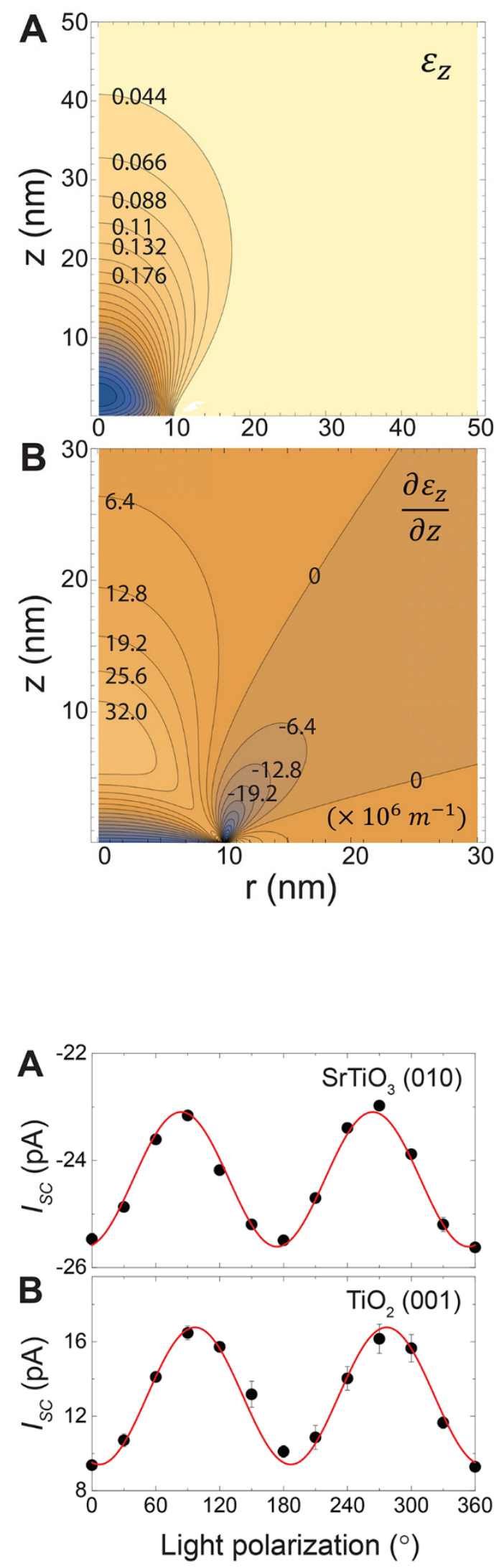

First release: 19 April 2018
Fig. 2. Spatial distributions of strain and strain gradient induced by an ideal spherical indenter. (A) The $z$-component of strain and (B) its partial derivative with respect to $z$. The contact area with a radius of $10 \mathrm{~nm}$ is centered at the origin, and the force is pointing upward. The positive (negative) value of the strain means a tensile (compressive) strain along the $z$-axis.
Fig. 3. Light polarization dependence of the force-induced photocurrent. The sinusoidal dependence of the photocurrent measured on (A) $\mathrm{SrTiO}_{3}(010)$ face and (B) $\mathrm{TiO}_{2}$ (001) face under $405 \mathrm{~nm}$ laser illumination. The red lines are the fits to Eq. 1. 

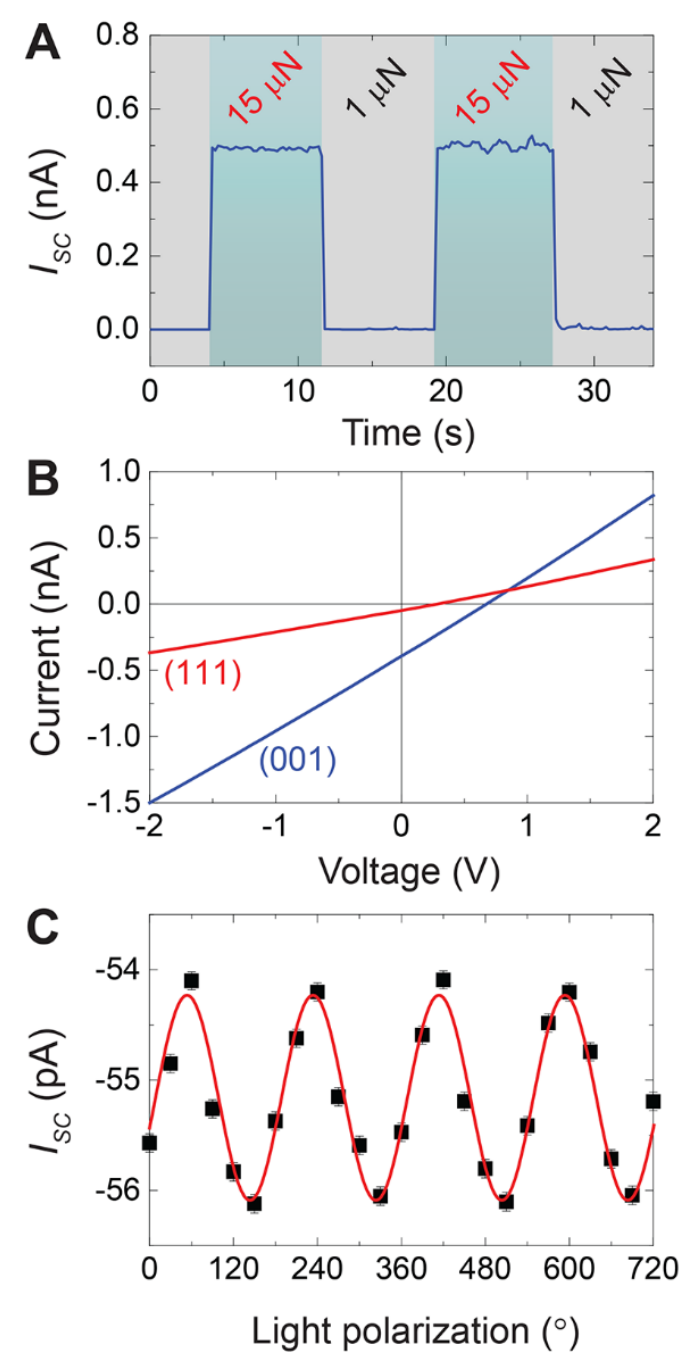

Fig. 4. Flexo-photovoltaic effect extended to covalent crystal and to the micrometer scale. (A) Force-induced photocurrent on (001) Si crystal measured by Ph-AFM under illumination of the top intended surface. (B) Current-voltage characteristics measured on (001) and (111) faces of a $\mathrm{SrTiO}_{3}$ crystal by the microindenter applying a $4 \mathrm{~N}$ force and illumination of the top intended surface. (C) The light polarization dependence of the photocurrent on $\mathrm{SrTiO}_{3}(010)$ face measured by the micro-indenter under illumination on the side surface. The red line is the fitting of experimental data with Eq. 1. Because the polarization angle origin of the microindenter is not coincident with the Ph-AFM setup, the oscillating Isc has a phase shift compared with Fig. 3B. 